\title{
From UML State Machines to code and back again!
}

\author{
Van Cam Pham, Ansgar Radermacher, Sébastien Gérard \\ CEA-List, Laboratory of Model-Driven Engineering for Embedded Systems (LISE) \\ Gif-sur-Yvette, France \\ Email: first-name.lastname@cea.fr
}

\begin{abstract}
UML state machines and their visual representations are much more suitable to describe logical behaviors of system entities than equivalent text based description such as IF-THEN-ELSE or SWITH-CASE constructions. Although many industrial tools and research prototypes can generate executable code from such a graphical language, generated code could be manually modified by programmers. After code modifications, round-trip engineering is needed to make the model and code consistent, which is a critical aspect to meet quality and performance constraints required for software systems. Unfortunately, current UML tools only support structural concepts for roundtrip engineering such as those available from class diagrams. In this paper, we address the round-trip engineering of UML statemachine and its related generated code. We propose an approach consisting of a forward process which generates code by using transformation patterns, and a backward process which is based on code pattern detection to update the original state machine model from the modified code. We implemented a prototype and conducted several experiments on different aspects of the roundtrip engineering to verify the proposed approach.
\end{abstract}

\section{INTRODUCTION}

$\mathbf{T}$ HE so-called Model-Driven Engineering (MDE) approach relies on two paradigms, abstraction and automation [1]. It is recognized as very efficient for dealing with complexity of today's systems. Abstraction provides simplified and focused views of a system and requires adequate graphical modeling languages such as Unified Modeling Language (UML). Even, if the latter is not the silver bullet for all software related concerns, it provides better support than text-based solutions for some concerns such as architecture and logical behavior of application development. UML state machines (USMs) and their visual representations are much more suitable to describe logical behaviors of system entities than any equivalent text based descriptions. The gap from USMs to system implementation is reduced by the ability of automatically generating code from USMs [2], [3], [4], [3].

Ideally, a full model-centric approach is preferred by MDE community due to its advantages [5]. However, in industrial practice, there is significant reticence [6] to adopt it. On one hand, programmers prefer to use the more familiar textual programming language. On the other hand, software architects, working at higher levels of abstraction, tend to favor the use of models, and therefore prefer graphical languages for describing the architecture of the system. The code modified by programmers and the model are then inconsistent. Roundtrip engineering (RTE) [7] is proposed to synchronize different software artifacts, model and code in this case [8]. RTE enables actors (software architect and programmers) to freely move between different representations [8] and stay efficient with their favorite working environment.

Unfortunately, current industrial tools such as for instance Enterprise Architect [9] and IBM Rhapsody[10] only support structural concepts for RTE such as those available from class diagrams and code. Compared to RTE of class diagrams and code, RTE of USMs and code is non-trivial. It requires a semantical analysis of the source code, code pattern detection and mapping patterns into USM elements. This is a hard task, since mainstream programming languages such as $\mathrm{C}++$ and JAVA do not have a trivial mapping between USM elements and source code statements.

For software development, one may wonder whether this RTE is doable. That is, why do the industrial tools not support the propagation of source code modifications back to original state machines? Several possible reasons to this lack are (1) the gap between USMs and code, (2) not every source code modification can be reverse engineered back to the original model, and (3) the penalty of using transformation patterns facilitating the reverse engineering that may not be the most efficient (e.g. a slightly larger memory overhead).

This paper addresses the RTE of USMs and object-oriented programming languages such as $\mathrm{C}++$ and JAVA. The main idea is to utilize transformation patterns from USMs to source code that aggregates code segments associated with a USM element into source code methods/classes rather than scatters these segments in different places. Therefore, the reverse direction of the RTE can easily statically analyze the generated code by using code pattern detection and maps the code segments back to USM elements. Specifically, in the forward direction, we extend the double dispatch pattern presented in [11]. Traceability information is stored during the transformations. We implemented a prototype supporting RTE of state-machine and $\mathrm{C}++$ code, and conducted several experiments on different aspects of the RTE to verify the proposed approach. To the best of our knowledge, our implementation is the first tool supporting RTE of SM and code.

To sum up, our contribution is as followings:

- An approach to round-tripping USMs and object-oriented code.

- A first tooling prototype supporting RTE of USMs and $\mathrm{C}++$ code

- An evaluation of the proposed approach including:

- An automatic evaluation of the proposed RTE approach with the prototype. 
- A lightweight evaluation of the semantic conformance of the runtime execution of generated code.

The remainder of this paper is organized as follows: Our proposed approach is detailed in Section II. The implementation of the prototype is described in Section III. Section IV reports our results of experimenting with the implementation and our approach. Section V shows related work. The conclusion and future work are presented in Section VI.

\section{APPROACH}

This section presents our RTE approach. At first, it sketches USM concepts supported by this study. The outline and the detail of the approach are presented afterward.

\section{A. Scope}

A USM describes the behavior of an active UML class which is called context class. A USM has a number of states and well-defined conditional transitions. A state is either an atomic state or a composite state that is composed of substates and has at most one active sub-state at a certain time. Transitions between states can be triggered by external or internal events. An action (effect) can also be activated by the trigger while transitioning from one state to another state. A state can have associated actions such as entry/exit/doActivity executed when the state is entered/exited or while it is active, respectively.

\section{B. Approach outline}

Our RTE approach is based on the double-dispatch pattern presented in [11] for mapping from USM to a set of classes with embedded code fragments. Fig. 1 shows the outline of our RTE approach consisting of a forward and a backward/reverse (engineering) process. In the forward process, a USM is transformed into UML classes in an intermediate model. The use of the intermediate model facilitates the transformation from the USM to code. Each class of the intermediate model contains attributes, operations and method bodies (block of text) associated with each implemented operation. The transformation utilizes several patterns which will be presented later.

When the source code is modified, a syntactic analysis process belonging to the backward transformation checks whether the modified code conforms to the USM semantics (see Subsection II-D3 for the detail of the analysis). This ${ }^{2}$ transformation takes as input the created intermediate model 2 and the USM to update these models sequentially. While the forward process can generate code from hierarchical and concurrent USMs, the backward one only works for hierarchical machines excluding pseudo-states which are history, join, fork, ${ }^{3}$ choice and junction. These features are in future work.

\section{From UML state machine to UML classes}

This section describes the forward process which utilizes transformation patterns for states, transitions, and events to an intermediate model and code. We start by a simple USM ${ }_{45}$

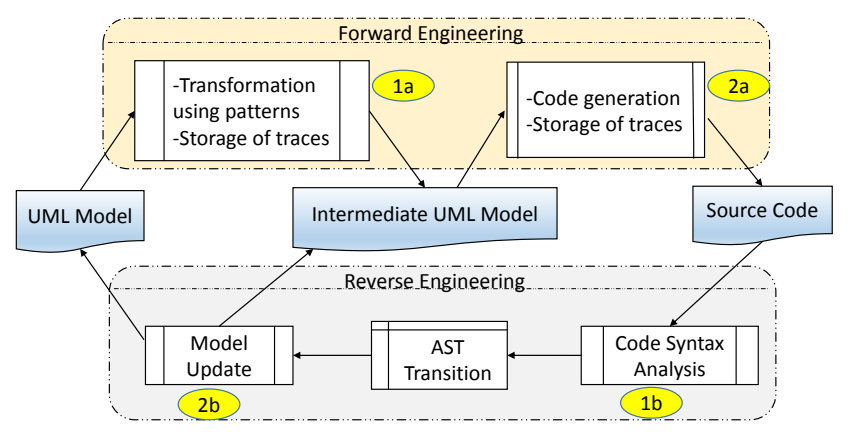

Fig. 1. Outline of state machine and code RTE

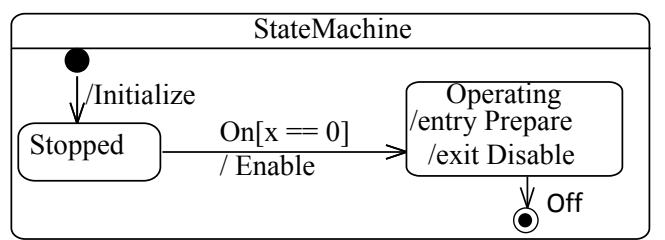

Fig. 2. An example of USM for tracing table

example in Fig. 2. It consists of two states (Stopped and Operating), two external events ( $O n$ and $O f f$ ), transitions, and an initial and a final pseudo state. Listing 1 shows a code portion generated from the USM following our approach.

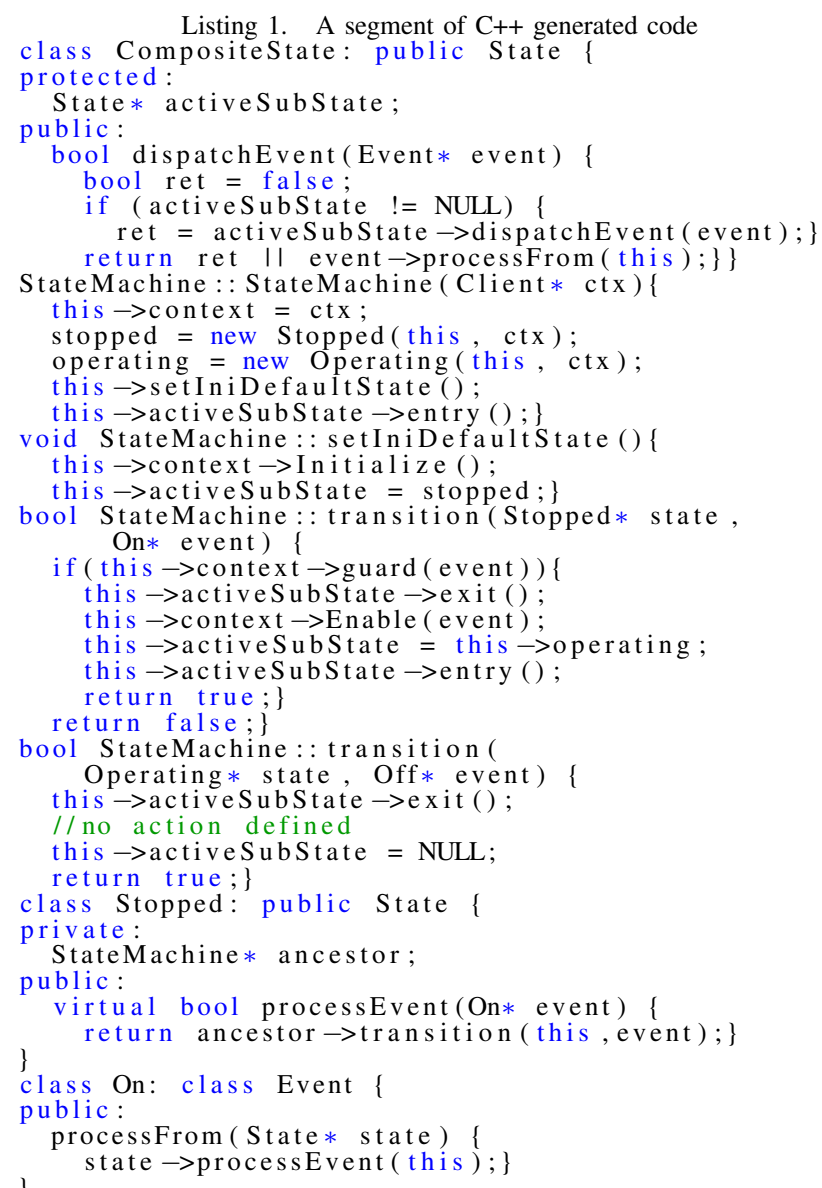




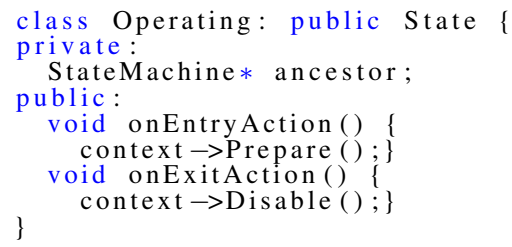

1) Transformation of states: Each state of the USM is transformed into a UML class. A state class inherits from a base class, namely, State (the detail of this class is not shown due to space limitation). State defines a reference to the context class, a process event operation for each event, state actions (entry/exit/doActivity). A bidirectional relationship is established between a state class and the state class associated with the containing state. For example, the USM example, considered as a composite state, has attributes typed by classes associated with its contained states, Stopped and Operating in Listing 1, lines 12-13. Inversely, attributes named ancestor (line 36) and typed by StateMachine in the classes Stopped and Operating are used to associate with the parent state.

A composite state class, which inherits from a base composite class (line 1), has an attribute activeSubState (line 3) indicating the active sub-state of the composite state and a dispatchEvent operation (line 5), which dispatches incoming events to the appropriate active state.

The dispatchEvent method implemented in the base composite state class delegates the incoming event processing to its active sub-state (line 8). If the event is not accepted by the active sub-state, the composite state processes it (line 9).

2) Transformation of events: Each event is transformed into a UML class (see lines 41-45 in Listing 1). An event can be either a CallEvent, SignalEvent or TimeEvent (see the UML specification for definitions of these events). An event class associated with a CallEvent inherits from a base event class and contains the parameters in form of attributes typed by the same types as those of the associated operation. The operation must be a member of the context class (a component as described above). For example, a call event CallEventSend associated with an operation named Send, which has two input parameters typed by Integer, is transformed into a class CallEventSend having two attributes typed by Integer. When a component receives an event, the event object is stored in an event queue.

A signal event enters the component through a port typed by the signal. The implementation view of this scenario depends on the mapping of component-based to object-oriented concepts. In the following, we choose the mapping done in Papyrus Designer [12]. In this mapping, the signal is transferred to the context class by an operation provided by the class at the associated port. Therefore, the transfer of a signal event becomes similar to that of CallEvent. For example, a signal event containing a data SignalData arrives at a port $p$ of a component $C$. The transformation derives an interface SignalDataInterface existing as the provided interface of $p$. SignalDataInterface has only one operation pushSignalData whose body will be generated to push the event to the event queue of the component. Therefore, the processing of a SignalEvent is the same as that of a CallEvent. In the following sections, the paper only considers CallEvent and TimeEvent.

A TimeEvent is considered as an internal event. The source state class of a transition triggered by a TimeEvent executes a thread to check the expiration of the event duration as in [13] and puts the time event in the event queue of the context class.

3) Transformation of transitions and actions: Each action is transformed into an operation in the transformed context class. Entry/Exit/doActivity actions have no parameters while transition actions and guards accept the triggering event object. doActivity is implicitly called in the State class and executed in a thread which is interrupted when the state changes.

OnEntryAction and OnExitAction - abstract methods in the base state class State - are called by the entry and exit methods, respectively. Lines 50-53 in Listing 1 show how these methods are overwritten by the Operating class. Prepare and Disable, implemented in the context class, are called in these methods, respectively.

A transition is transformed into an operation taking as input the source state object and the event object similarly to DD. Transitions transformed from triggerless transition which has no triggering events accept only the source state object as a parameter. For example, the Enable action in the example is created in the context class and called by the transition method in lines 19-26. The guard guard is implemented as a method in the context class and called in line 21 .

\section{Reverse engineering from code to USM}

This section describes the backward process.

1) Method Overall: The overall method for backward transformation is shown in Fig. 3. The modified code is first analyzed by partly inspecting the code syntax and semantics to guarantee that it is reversible. There are cases in which not all code modifications can be reversed back to the USM. The analysis also produces an output (output2) whose format is described later. If the intermediate model or the original USM is absent (the lower part of Fig. 3), a new intermediate model and a new USM are created from the UML model. In the contrary, the previous code taken, for instance, from control versioning systems is also semantically analyzed to have its output (output1) (the upper part of Fig. 3). Output1 and Output 2 are then compared with each other to detect actual semantic changes which are about to be propagated to the original model.

Due to space limitation, we only show how to reconstruct (create) a new USM from the modified code.

2) Illustration example: To give an overview how the backward works, Fig. 4 presents a partition for mapping from the code segments generated from the example in Fig. 2 to actual USM concepts. Each partition consists of a code segment and the corresponding model element which are mapped in the backward direction. For example, the Stopped class in 


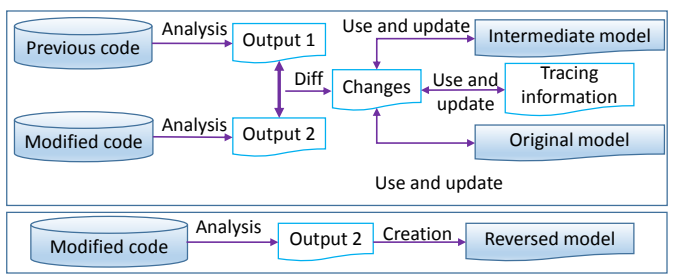

Fig. 3. Overall method for reversing code to state machine

code is mapped to the state Stopped of the state machine. The method transition is mapped back to the corresponding transition whose source state and triggering event are the input parameters' types of the method.

3) Semantic Analysis: The output of the semantic analysis contains a list of event names, a list of state names, a list of transitions in which each has a source state, a target state, a guard function, an action function and an event represented in so called abstract syntax tree (AST) transition [15]. For example, Fig. 5 presents the EMF [14] representation of transitions in a $\mathbf{C + +}$ AST in which IStructure and IFunctionDeclaration represent a structure and a function in $\mathbf{C + +}$, respectively. Each state name is also associated with an ancestor state, an entry action, an exit action, a default sub-state and a final state. The output is taken by analyzing the AST. The analysis process consists of recognizing different patterns. Table I shows the main patterns including state, transition and event.

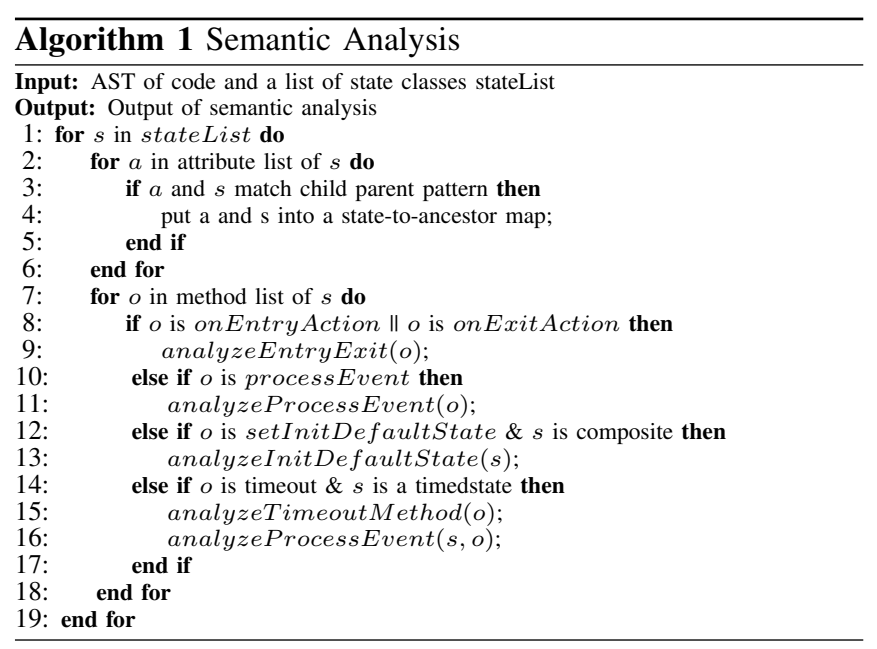

Algorithm 1 shows the algorithm used for analyzing code semantics. Due to space limitation, analyzeEntryExit, analyzeProcessEvent, analyzeInitDefaultState, analyzeTimeoutMethod and analyzeProcessEvent are not presented but they basically follow the pattern description as above. In the first step of the analysis process, for each state class, it looks for an attribute typed by the state class, the class containing the attribute then becomes the ancestor class of the state class. The third steps checks whether the state class has an entry or exit action by looking for the implementation of the onEntryAction or onExitAction, respectively, in the state class to recognize the Entry/Exit action pattern. Consequently, event processing, initial default state of composite state and time event patterns are detected following the description as above.

4) Construction of USM from analysis output: If an intermediate model is not present, a new intermediate model and a new USM are created by a reverse engineering and transformation from the output of the analysis process. The construction is straightforward. At first, states are created. Secondly, UML transitions are built from the AST transition list. Lastly, action/guard/triggering event of a UML transition is created if the associated AST transition has these.

For example, assuming that we need to adjust the USM example shown in Fig. 2 by adding a guard to the transition from Operating to the final state. The adjustment can be done by either modifying the USM model or the generated code. In case of modifying code, the associated transition function in Listing 1 is edited by inserting an if statement which calls the guard method implemented in the context class. The change detection algorithm adds the transition function into the updated list since it finds that the source state, the target state and the event name of the transition is not changed. By using mapping information in the mapping table, the original transition in the USM is retrieved. The guard of the original transition is also created.

\section{IMPLEMENTATION}

The proposed approach is implemented in a prototype existing as an extension of the Papyrus modeler [15]. Each USM is created by using the state machine diagram implemented by Papyrus to describe the behavior of a UML class. Lowlevel USM actions are directly embedded in form of a block of code written in specific programming languages such as C++/JAVA into the USM. C++ code is generated by the prototype but other object-oriented languages can be easily generated. The code generation consists of transforming the USM original containing the state machine to UML classes and eventually to code by a code generator following the proposed approach. The code generator can generate code for hierarchical and concurrent USMs. In the reverse direction, code pattern detection is implemented as described in the previous section to analyze USM semantics. If the generated code is modified, two options are supported by the prototype to make the USM and code consistent again. One is to create a new model containing the USM from the modified code in the same Eclipse project and the other one is to update the original USM by providing as input the intermediate model and the original model. At the writing moment, the prototype does not support the reverse of concurrent USMs and pseudo states, which are history, join, fork,choice, and junction.

\section{EXPERIMENTS}

In order to evaluate the proposed approach, we answer three questions stated as followings.

RQ1: A state machine $s m$ is used for generating code. The generated code is reversed by the backward transformation to produce another state machine $s m$ '. Are $s m$ and $s m$ ' identical? 


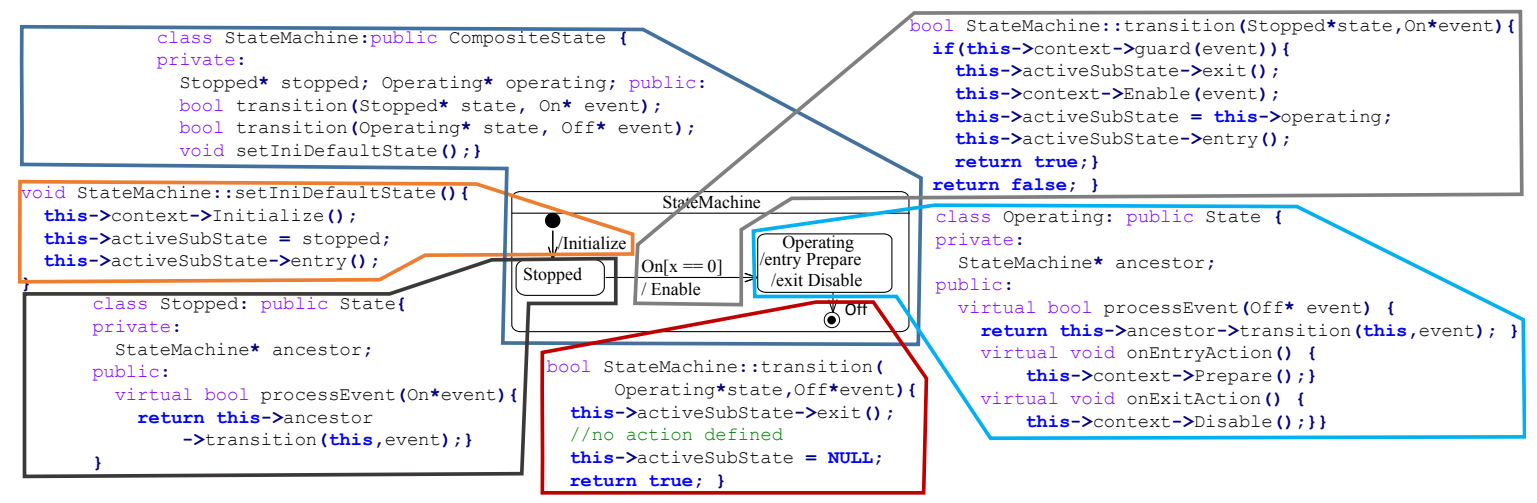

Fig. 4. USM element-code segment mapping partition

TABLE I

PATTERN RECOGNITION FOR REVERSE ENGINEERING GENERATED CODE

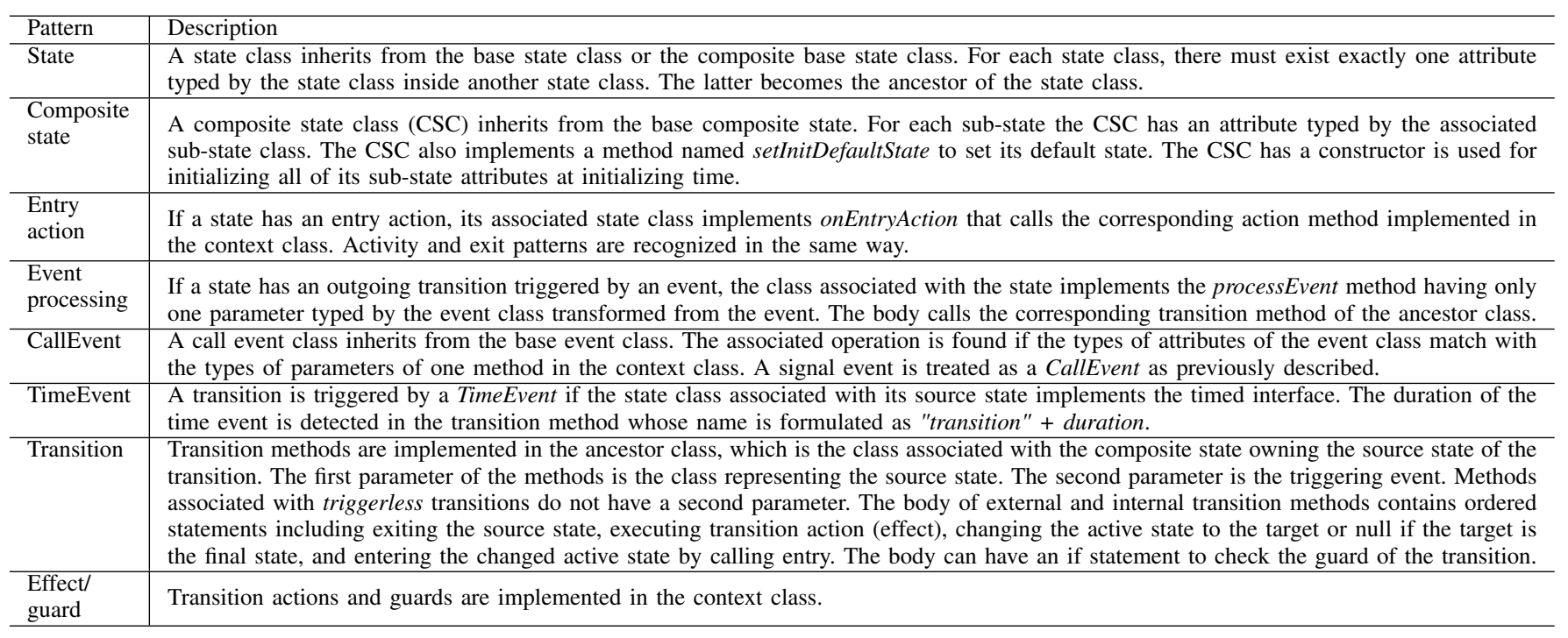

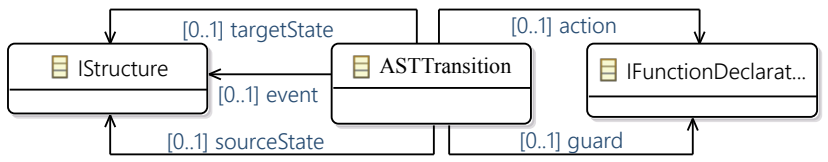

Fig. 5. Transitions output from the analysis

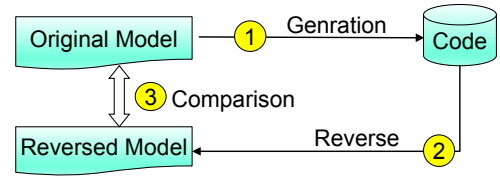

Fig. 6. Evaluation methodology to answer RQ1
In other words: whether the code generated from USMs model can be used for reconstructing the original model. This question is related to the GETPUT law defined in [16].

RQ2: RQ1 is related to the static aspect of generated code. RQ2 targets to the dynamic aspect. In other words, whether the runtime execution of code generated from USMs by the generator is semantic-conformant [17]?

This section reports our experiments targeting the three questions. Two types of experiments are conducted and presented in Subsections IV-A and IV-B, respectively.

\section{A. Reversing generated code}

This experiment is targeting RQ1. Fig. 6 shows the experimental methodology to answer RQ1. The procedure for this experiment, for each original UML model containing a state machine, consists of 3 steps: (1) code is generated from an original model, (2) the generated code is reversed to a reversed model, and (3) the latter is then compared to the original state machine.

Random models containing hierarchical state machines are automatically generated by a configurable model generator. The generator can be configured to generate a desired average number of states and transitions. For each model, a context 
TABLE II

THREE OF MODEL RESULTS OF GENERATION AND REVERSE: ABBREVIATIONS ARE ATOMIC STATES (AS), COMPOSITE STATES (CS), TRANSITIONS (T), CALL EVENTS (CE), TIME EVENTS (TE)

\begin{tabular}{|l|l|l|l|l|l|l|}
\hline Test ID & AS & CS & T & CE & TE & Is reverse correct? \\
\hline 1 & 47 & 33 & 234 & 145 & 40 & Yes \\
\hline 2 & 42 & 38 & 239 & 145 & 36 & Yes \\
\hline.. &.. &.. &.. &.. &.. & Yes \\
\hline 300 & 41 & 39 & 240 & 142 & 37 & Yes \\
\hline
\end{tabular}

class and its behavior described by a USM are generated. Each USM contains 80 states including atomic and composite states, more than 234 transitions. The number of lines of generated $\mathrm{C}++$ code for each machine is around 13500. Names of the generated states are different. An initial pseudo state and a final state are generated for each composite state and containing state machine. Other elements such as call events, time events, transition/entry/exit actions and guards are generated with a desired configuration. For each generated call event, an operation is generated in the context class which is also generated. The duration is generated for each time event.

Table II shows the number of several types of elements in the generated models, including the comparison results, for 3 of the 300 models created by the generator. We limited ourselves to 300 models for practical reasons. No differences were found during model comparison. The results of this experiment show that the proposed approach and the implementation can successfully do code generation from state machines and reverse.

\section{B. Semantic conformance of runtime execution}

a) Bisimulation for semantic-conformance: To evaluate the semantic conformance of runtime execution of generated code, we use a set of examples provided by Moka [18]. Moka is a model execution engine offering Precise Semantics of UML Composite Structures [17]. Fig. 7 shows our method. We first use our code generator to generate code (Step (1)) from the Moka example set. Step (2) simulates the examples by using Moka to extract the sequence (SimTraces) of observed traces including executed actions. The sequence (RTTraces) of traces is also obtained by the runtime execution of the code generated from the same state machine in a Step (3). The generated code is semantic-conformant if the sequences of traces are the same for both of the state machine and generated code [19]. The current version of Moka does not support simulation for TimeEvent and history pseudo states, we therefore leave experiments for TimeEvent as future work.

For example, Fig. 8 (a) shows a USM example with triggerless transitions (autotransitions) T3. The USM contains two states, Waiting, which is the initial state, and Incrementing, which increases an integer number from 0 to 5 by using the effect of T3. The latter also has a guard checking whether the number is less than 5 . The increase is executed after the USM receives an event named start to transition the initial state Waiting to Incrementing. Suppose that executions of the effects of $T 3$ and $T 4$ produce traces $<T 3>$ and $<T 4>$ (by using

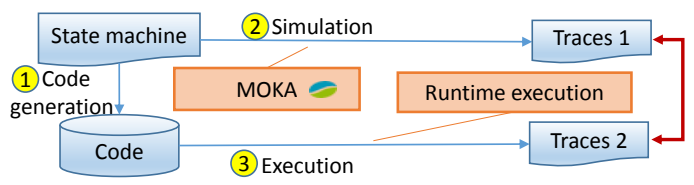

Fig. 7. Semantic conformance evaluation methodology
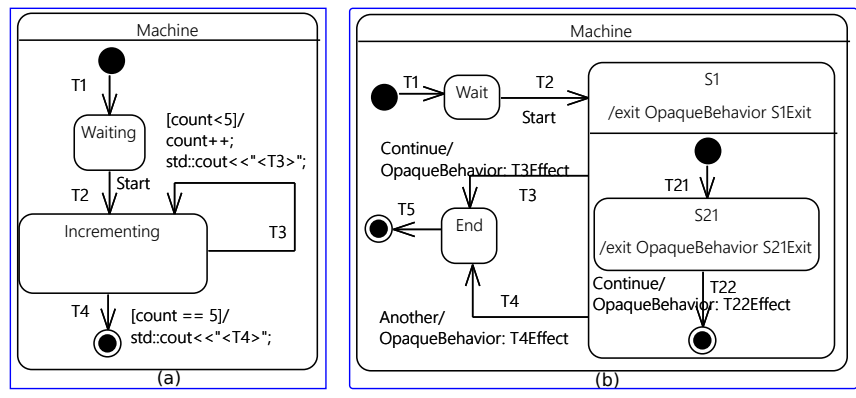

Fig. 8. Self-triggerless transition and event processing example

MOKA, e.g.), respectively. Due to the guard of $T 3$, the effect of $T 3$ is executed five times followed by an execution of the effect of T4. After the completion of the USM, the obtained sequence of traces is $\langle T 3\rangle<T 3\rangle<T 3\rangle<T 3\rangle<T 3\rangle<T 4\rangle$ (since the Incrementing state does not have an entry, exit, or a doActivity, only the transition effect $T 3$ produces traces). The sequence RTTraces obtained by the runtime execution must be equivalent. RTTraces is obtained by simply printing logging information for each action (effect).

Within our scope as previously defined 30 examples of the Moka example set are tested. SimTraces and RTTraces for each case are the same. This indicates that, within our study scope, the runtime execution of code generated by our generator can produce traces semantically equivalent to those obtained via simulation.

After experimenting with our code generator, we compare our results to the observed traces obtained by executing code generated Umple [20]. We find that the obtained traces in case of Umple are not UML-compliant in triggerless transitions and some cases of event processing. Specifically, for the example in Fig. 8 (a), code generated by Umple only produces $<T 3>$ as the trace sequence. Umple does not support events which are accepted by sub-states and the corresponding composite state as in Fig. 8 (b) in which both $S 1$ and $S 21$ accept the event Continue. As the processing event example in Fig. 8 (b), assuming that there is an event Continue incoming to the state machine which has a current configuration ( $S 1$, S21) as current active states. While, according to the UML specification, the incoming event should be processed by the inner states of the active composite/concurrent state if the inner states accept it, otherwise the parent state does. Therefore, the next configuration should be (S1, final state) and the T22Effect effect of the transition $T 22$ should be executed.

b) Finite state machine: In addition to the experiment using MOKA, we evaluate the semantic-conformance by using deterministic finite state machines (FSMs). The latter is a 


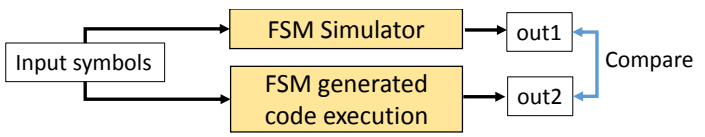

Fig. 9. FSM experiment method

mathematical model of computation and also a simplified version of UML state machine. In this experiment, we use FSMs for recognizing input symbols. Each FSM contains many atomic states. The active state of the FSM can be changed following the acceptance of an input symbol. Fig. 9 shows our method to experiment. For each FSM, we create an equivalent USM. Each input symbol of the FSM is considered as an event of the USM. We use the FSM simulator in [21] to generate and simulate FSMs. For each FSM, a list of observed states is recorded as output (outl) of the simulation for each symbol list. The latter is also the input of the generated code runtime execution of the equivalent USM which produces an output out 2 . We then compare out1 and out 2 .

We limit the number of FSMs to 20 and the number of symbol list for each FSM to 30 for practical concerns. 600 sequences of states obtained by the simulation and a same number of sequences taken by the runtime execution are respectively compared and found being equal. This results that our code generation approach can produce semanticconformant code in case of FSM.

\section{RELATED WORK}

Our work is motivated by the desire to reduce the gap between and synchronize artifacts at different levels of abstraction, model and code in particular, in developing reactive systems. Specifically, the usage of USMs for describing the logical behavior of complex, reactive systems is indispensable. In the following sections, we compare our approach to related topics recorded in the literature.

\section{A. Implementation and code generation techniques for USMs}

Implementation and code generation techniques for USMs are closely related to the forward engineering of our RTE.

Switch/if is the most intuitive technique implementing a "flat" state machine. The latter can be implemented by either using a scalar variable [2] and a method for each event or using two variables as the current active state and the incoming event used as the discriminators of an outer switch statement to select between states and an inner one/if statement, respectively. The double dimensional state table approach [3] uses one dimension represents states and the other one all possible events. The behavior code of these techniques is put in one file or class. This practice makes code cumbersome, complex, difficult to read and less explicit when the number of states grows or the state machine is hierarchical. Furthermore, these approaches requires every transition must be triggered by at least an event. This is obviously only applied to a small sub-set of USMs.
State pattern [4], [3] is an object-oriented way to implement flat state machines. Each state is represented as a class and each event as a method. Separation of states in classes makes the code more readable and maintainable. This pattern is extended in [22] to support hierarchical-concurrent USMs. However, the maintenance of the code generated by this approach is not trivial since it requires many small changes in different places.

Many tools, such as [10], [9], apply these approaches to generate code from USMs. Readers of this paper are recommended referring to [23] for a systematic survey on different tools and approaches generating code from USMs.

Double-dispatch (DD) pattern in [11] in which represent states and events as classes. Our generation approach relies on and extends this approach. The latter profits the polymorphism of object-oriented languages. However, DD does not deal with triggerless transitions and different event types supported by UML such as CallEvent, TimeEvent and SignalEvent. Furthermore, DD is not a code generation approach but an approach to manually implementing state machines.

\section{B. Round-trip engineering}

Our RTE is related to synchronization of model-code and models themselves that a large number of approaches support. This paper only shows the most related approaches.

\section{Model-code synchronization}

Commercial and open-source tools such as [9], [10] only support RTE of architectural model elements and code. Systematic reviews of some of these tools are available in [24].

Some RTE techniques restrict the development artifact to avoid synchronization problems. Partial RTE and protected regions [25] aim to preserve code changes which cannot be propagated to models. This approach separates the code regions that are generated from models from regions which are allowed to be edited by developers. This form of RTE is unidirectional only and does not support iterative development [26] Our approach does not separate different regions but supports a semantic code analysis in the reverse direction.

Fujaba [27] offers an RTE environment. An interesting part of Fujaba is that it abstracts from Java source code to UML class diagrams and a so-called story-diagrams. Java code can also be generated from these diagrams. RTE of these diagrams and code works but limited to the naming conventions and implementation concepts of Fujaba which are not UML-compliant.

\section{Model synchronization}

RTE of models is tackled by many approaches categorized by its model transformation from total, injective, bi-directional to partial non-injective transformations [7]. Techniques and technologies, such as Triple Graph Grammar (TGG) [28] and QVT-Relation [29], allow synchronization between source and target elements that have non-injective mappings. These techniques require a mapping model to connect the source and target models which need to be persisted in a model store 
[30]. Mappings between USMs and code in our approach are non-bijective. We only use simple tables for storing tracing information.

Differently from other approaches, ours is specific to RTE of USMs and code. The goal is to provide a full modelcode synchronization supporting for rapidly, iteratively, and efficiently developing reactive systems.

\section{CONCLUSiON}

This paper presented a novel approach to RTE from USMs to code and back. The forward process of the approach is based on different patterns transforming USM elements into an intermediate model containing UML classes. Object-oriented code is then generated from the intermediate model by existing code generators. In the backward direction, code is analyzed and transformed into an intermediate whose format is close to the semantics of USMs. USMs are then reconstructed from the intermediate format.

The paper also showed the results of several experiments on different aspects of the proposed RTE with the tooling prototype. Specifically, the experiments on the correctness and semantic conformance of code using the proposed RTE are conducted. Although, the reverse direction only works if manual code is written following pre-defined patterns, the semantics of USMs is explicitly present in generated code and easy to follow/modify.

While the semantic conformance of code generated is critical, the paper only showed a lightweight experiment on this aspect. A systematic evaluation is therefore in future work. We will also compare our code generation approach with commercial tools such as Rhapsody and Enterprise Architect. Furthermore, as evaluated in [7], the approach inheriting from the double-dispatch trades a reversible mapping for a slightly larger overhead. For the moment, the approach does not support RTE of concurrent state machines and several pseudostates. Hence, future work should resolve these issues.

\section{ACKNOWLEDGMENT}

The work presented in this paper is supported by the European project SafeAdapt, grant agreement No. 608945, see http://www.SafeAdapt.eu. The project deals with adaptive system with additional safety and real time constraints. The adaptation and safety aspects are stored in different artifacts in order to achieve a separation of concerns. These artifacts need to be synchronized.

\section{REFERENCES}

[1] G. Mussbacher, D. Amyot, R. Breu, J.-m. Bruel, B. H. C. Cheng, P. Collet, B. Combemale, R. B. France, R. Heldal, J. Hill, J. Kienzle, and M. Schöttle, "The Relevance of Model-Driven Engineering Thirty Years from Now," ACM/IEEE 17th International Conference on Model Driven Engineering Languages and Systems (MODELS), pp. 183-200, 2014.

[2] G. Booch, J. Rumbaugh, and I. Jacobson, The Unified Modeling Language User Guide, 1998, vol. 3.

[3] B. P. Douglass, Real-time UML : developing efficient objects for embedded systems, 1999.
[4] A. Shalyto and N. Shamgunov, "State machine design pattern," Proc. of the 4th International Conference on.NET Technologies, 2006.

[5] B. Selic, "What will it take? a view on adoption of model-based methods in practice," Software \& Systems Modeling, vol. 11, no. 4, pp. 513-526, 2012.

[6] J. Hutchinson, M. Rouncefield, and J. Whittle, "Model-driven engineering practices in industry," in Proceedings of the 33rd International Conference on Software Engineering, ser. ICSE '11. New York, NY, USA: ACM, 2011, pp. 633-642.

[7] T. Hettel, M. Lawley, and K. Raymond, "Model synchronisation: Definitions for round-trip engineering," in Lecture Notes in Computer Science (including subseries Lecture Notes in Artificial Intelligence and Lecture Notes in Bioinformatics), vol. 5063 LNCS, 2008, pp. 31-45.

[8] S. Sendall and J. Küster, "Taming model round-trip engineering," Proceedings of Workshop Best Practices for Model-Driven Software Development, p. 13, 2004.

[9] SparxSystems, "Enterprise Architect," Sep. 2016. [Online]. Available: http://www.sparxsystems.eu/start/home/

[10] IBM, "Ibm Rhapsody." [Online]. Available: http://www.ibm.com/ developerworks/downloads/r/rhapsodydeveloper/

[11] V. Spinke, "An object-oriented implementation of concurrent and hierarchical state machines," Information and Software Technology, vol. 55 no. 10 , pp. 1726-1740, Oct. 2013.

[12] "Papyrus Designer." [Online]. Available: https://wiki.eclipse.org/ Papyrus_Designer

[13] I. Niaz and J. Tanaka, "Mapping UML statecharts to java code." IASTED Conf. on Software Engineering, pp. 111-116, 2004.

[14] R. Gronback, "Eclipse Modeling Project." [Online]. Available: http://www.eclipse.org/modeling/emf/

[15] CEA-List, "Papyrus Homepage Website," https://eclipse.org/papyrus/.

[16] J. N. Foster, M. B. Greenwald, J. T. Moore, B. C. Pierce, and A. Schmitt, "Combinators for Bidirectional Tree Transformations: A Linguistic Approach to the View-update Problem," ACM Trans. Program. Lang. Syst., vol. 29, no. 3, May 2007.

[17] OMG, "Precise Semantics Of UML Composite Structures," no. October, 2015 .

[18] "Moka Model Execution." [Online]. Available: https://wiki.eclipse.org/ Papyrus/UserGuide/ModelExecution

[19] J. O. Blech and S. Glesner, "Formal verification of java code generation from uml models," in ... of the 3rd International Fujaba Days, 2005, pp. $49-56$.

[20] O. Badreddin, T. C. Lethbridge, A. Forward, M. Elasaar, and H. Aljamaan, "Enhanced Code Generation from UML Composite State Machines," Modelsward 2014, pp. 1-11, 2014.

[21] F. Simulator, "FSM Simulator," http://ivanzuzak.info/noam/webapps/ fsm_simulator/.

[22] I. A. Niaz, J. Tanaka, and others, "Mapping UML statecharts to java code." in IASTED Conf. on Software Engineering, 2004, pp. 111-116.

[23] E. Domínguez, B. Pérez, A. L. Rubio, and M. A. Zapata, "A systematic review of code generation proposals from state machine specifications," pp. 1045-1066, 2012.

[24] D. Cutting and J. Noppen, "An Extensible Benchmark and Tooling for Comparing Reverse Engineering Approaches," International Journal on Advances in Software, vol. 8, no. 1, pp. 115-124, 2015. [Online]. Available: https://ueaeprints.uea.ac.uk/53612/

[25] D. Frankel, Model Driven Architecture: Applying MDA to Enterprise Computing. New York, NY, USA: John Wiley \& Sons, Inc., 2002.

[26] S. Jörges, "Construction and evolution of code generators: A modeldriven and service-oriented approach," Lecture Notes in Computer Science (including subseries Lecture Notes in Artificial Intelligence and Lecture Notes in Bioinformatics), pp. 1-265, 2013.

[27] T. Kleín, U. A. Nickel, J. Niere, and A. Zündorf, "From uml to java and back again," University of Paderborn, Paderborn, Germany, Tech. Rep. tr-ri-00-216, September 1999.

[28] H. Giese and R. Wagner, "Incremental model synchronization with triple graph grammars," in Model Driven Engineering Languages and Systems. Springer, 2006, pp. 543-557.

[29] Q. Omg, "Meta Object Facility ( MOF ) 2 . 0 Query / View / Transformation Specification," Transformation, no. January, pp. 1-230, 2008.

[30] G. Bergmann, I. Ráth, G. Varró, and D. Varró, "Change-driven model transformations: Change (in) the rule to rule the change," Software and Systems Modeling, vol. 11, no. 3, pp. 431-461, 2012. 